\title{
A Segmentation based Channel Scheduling Scheme in Optical Burst Switching Networks
}

\author{
A. Mandloi \\ Electronics Engg Department \\ S.V.N.I.T Surat \\ Gujarat- India
}

\author{
V. Mishra \\ (Senior MIEEE) \\ Electronics Engg Department \\ S.V.N.I.T Surat \\ Gujarat- India
}

\begin{abstract}
Optical Burst Switching (OBS) is considered to be a promising paradigm for bearing IP traffic in Wavelength Division Multiplexing (WDM) optical networks. Scheduling of data burst in data channels in an optimal way is one of a key problem in Optical Burst Switched networks. The main concerns in this paper is to schedule the incoming bursts in proper data channel such that more burst can be scheduled so burst loss will be less. There are different algorithms exists to schedule data burst on data channels. Non-preemptive DelayFirst Minimum Overlap Channel with Void Filling and Nonpreemptive Segment-First Minimum Overlap Channel with Void Filling are best among other existing segmentation based void filling algorithms. Though it gives less burst loss but not existing the channel utilization efficiently. In this paper we introduce a new approach, which will give less burst loss and also utilize existing channels in efficient way. Also analyze the performance of this proposed scheduling scheme and compare it with the existing void filling algorithms. It is shown by simulations that the proposed scheme gives some better performances compared to the existing schemes.
\end{abstract}

\section{General Terms}

Core Node OBS, Scheduling, Segmentation based Void Filling Algorithm, Wavelength Conversion.

\section{Keywords}

Channel Utilization, FFUC-VF, LAUC, Horizon void filling, Channel Scheduling, non Preemptive Algorithms

\section{INTRODUCTION}

Optical burst switching (OBS) is emerging as the switching technology for next generation optical networks. Advantages of optical packet switching and circuit switching are combined in OBS and overcoming their limitations. Data (or payload) is separated from control packet. A control packet is sent before the payload to reserve the resources on the path to the destination of payload. When a control packet arrives at an intermediate node a wavelength scheduling algorithm is used by the scheduler to schedule the data burst on an outgoing wavelength channel. The required information to schedule a data burst is arrival time and duration of data burst, which are obtained from control packet. On the other hand, scheduler keeps availability of time slots on every wave length channel and schedule a data burst in a channel depending upon the scheduling algorithm it uses. Different scheduling algorithms have been proposed in literature to schedule payload/ data burst. They differ in burst loss and complexity. Depending upon the channel selection strategy, they can be classified as Horizon and Void filling algorithm. Horizon algorithm considers the channels which have no scheduled data burst at or after current time $t$ and the channels are called Horizon channels. Void filling algorithms consider the channels which have unused duration in between two scheduled data bursts. These are called Void channels. The example of non segmentation Horizon algorithms are FFUC, LAUC and non segmentation Void algorithms are FFUC-VF [3], LAUC-VF $[4,5,6,7]$ and Min-EV [8]. The example of segmentation Horizon algorithms are Non preemptive Minimum Overlap Channel (NP-MOC) [9], Non-preemptive Delay-First Minimum Overlap Channel (NP DFMOC) [9] and Nonpreemptive Segment-First Minimum Overlap Channel (NPSFMOC) [9]. And the examples of non segmentation void filling algorithms are Non preemptive Minimum Overlap Channel with Void Filling (NP-MOC-VF) [9] Nonpreemptive Delay-First Minimum Overlap Channel with Void Filling (NP-DFMOC-VF) [9] and Non-preemptive Segment First Minimum Overlap Channel with Void Filling (NPSFMOC-VF) [9]. Horizon algorithms are easy to implement and burst loss ratio is high. Whereas burst loss ratio is lower in Void filling algorithms but complex switching are required to implement. All, LAUC-VF, Min-EV, NPMOC-VF, NPDFMOC and NP-SFMOC-VF consider one side of a void [10]. There may be a possibility, in which a smaller data burst will be scheduled in a larger void where as a bigger data burst will be dropped. This will lead to higher burst blocking and lower channel utilization. In this chapter we propose a new channel scheduling algorithm which attempts to make efficient utilization of existing void within a channel. Thus, giving rise to higher channel utilization and lower blocking probability. Rest of the paper is organized as follows. Section 2 explains the existing scheduling algorithms. Limitations of the existing void filling algorithms are explained in Section 3. Working of the newly introduced scheme is explained Section 4. We compare our proposed scheme with NP-DFMOC-VF and NP-SFMOC-VF algorithms. Comparison and simulation results are presented in Section 5 


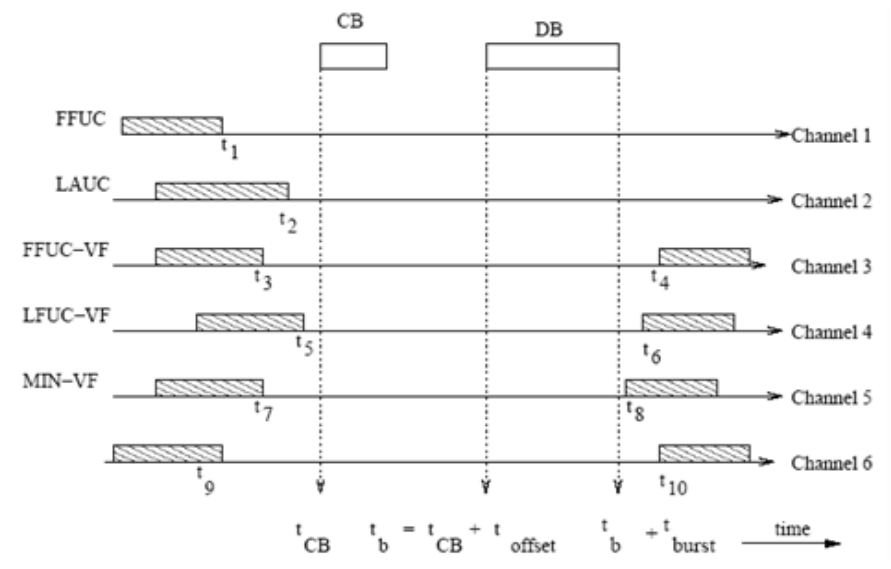

Fig 1: Illustration of Channel Scheduling in OBS

\section{CHANNEL SCHEDULING IN OBS}

When a control packet arrives at a core node, a wavelength channel scheduling algorithm is used to determine a wavelength channel on an outgoing link for the corresponding data burst. The information required by the scheduler such as the expected arrival time of the data burst and its duration are obtained from the control packet. The scheduler keeps track of the availability of time slots on every wavelength channel. It selects one among several idle channels. The selection of wavelength channel needs to be done in an efficient way so as to reduce the burst loss. At the same time, the scheduler must be simple and should not use any complex algorithm, because the routing nodes operate in a very high-speed environment handling a large amount of burst traffic. A complex scheduling algorithm may lead to the early data burst arrival situation wherein the data burst arrives before its control packet is processed and eventually the data burst is dropped

In this section we discuss various scheduling algorithms proposed in literature. These algorithms differ in their complexity and performance in terms of burst loss. A wavelength channel is said to be unscheduled at time $t$ when no data burst is using the channel at or after time t. Algorithms which consider unscheduled channels are called Horizon algorithm. A channel is said to be unused for the duration of voids between two successive data bursts and after the last data burst assigned to the channel. Algorithms which consider voids within channels are called void filling algorithm. According to scheduling strategy used scheduling algorithms can be classified as follows:

\section{- $\quad$ Horizon or without void filing. \\ - With void filling.}

Before going to the segmentation part algorithms is it first needed to know about horizon and void filling concept and for simpler we take the concept of non- segmentation algorithms to define that. In Figure 1, control packet arrives at a node at time $t_{\mathrm{CB}}$. Duration of payload is $\mathrm{t}_{\mathrm{burst}}$ and the offset time for the data burst $t_{\text {offset. }}$ The offset time is calculated as:

$\mathrm{t}_{\text {offset }}=\mathrm{H} * \Delta$

where $H$ is number of hops from source to destination and $\Delta$ is the time required for processing and switching the control packet. The time at which the first bit of payload arrives at the node is $\mathrm{t}_{\mathrm{CB}}+\mathrm{t}_{\mathrm{offset}}$ and the last bit arrive at $\mathrm{t}_{\mathrm{CB}}+\mathrm{t}_{\mathrm{offset}}+$ $\mathrm{x}$

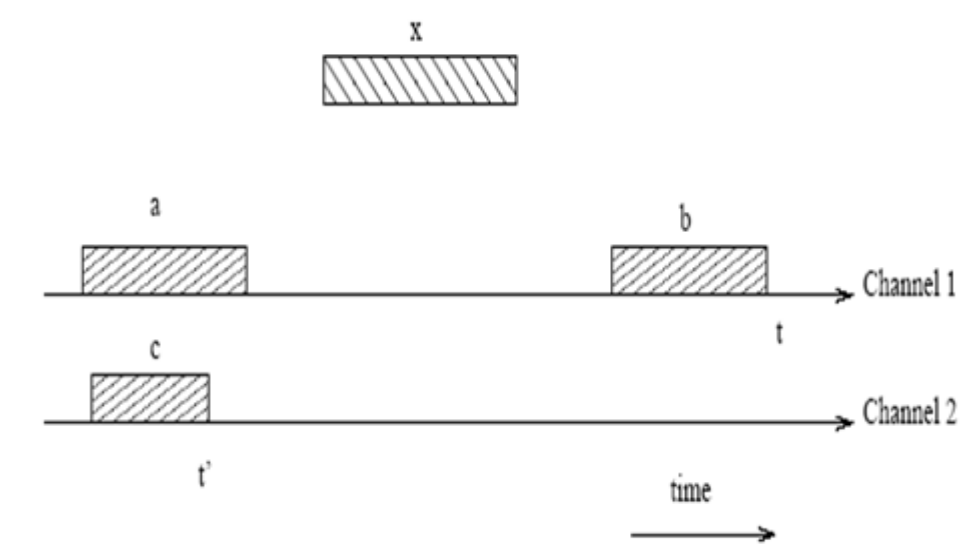

Fig 2: A scheduling Scenario

$t_{\text {burst }}$. We define unscheduled channel and void channel as following (see Figure 1)

Unscheduled channel: A wavelength channel is said to be unscheduled at time $t$ when no data burst is using the channel at or after $\mathrm{t}$.

Void channel: If a channel is unused for duration between two successive data bursts.

\section{LIMITATIONS OF EXISTING ALGORITHMS}

\subsection{Limitations of Horizon and Void Filling Algorithms}

Horizon scheduling algorithms consider the unscheduled channels to schedule a data burst. It does not consider the availability of void within a channel, which could otherwise be used in channel scheduling. For example consider the Figure 2. In this figure there two data bursts $a$ and $b$ are scheduled on channel 1 and data burst $c$ on channel 2. For horizon scheduling algorithms, channel 1 is available at time instant $\mathrm{t}$ and channel 2 is at $\mathrm{t}$ '. Suppose a data burst $\mathrm{x}$ arrives. Horizon scheduling algorithms will schedule the data burst $\mathrm{x}$ on channel 2 as shown in (see Figure 3). They do not consider the voids within a channel. In channel 1 there exist a void between data bursts $\mathrm{a}$ and $\mathrm{b}$ within which the data burst $\mathrm{x}$ could have been scheduled. Thus, horizon scheduling algorithms are not efficient in terms of channel utilization and gives rise to higher burst loss. On the other hand, void filling algorithms consider both unscheduled and void channel to schedule data bursts. For the scenario as shown in Figure 2, void filling algorithms will schedule data burst $\mathrm{x}$ on channel 1 (see Figure 4). Thus, increases the channel utilization. Any data burst arriving between t' and t could be schedule on channel 2, which otherwise could have been dropped in horizon algorithms. Thus horizon scheduling algorithms are not efficient in terms of burst loss and channel utilization in comparison to void filling algorithms.

\subsection{Limitations of Segmentation Based Algorithms}

Though void filling algorithms are efficient than horizon scheduling algorithms, but they are not the optimal scheduling algorithms. The limitations of void filling algorithms for nonsegmented and segmented are they lies in the fact that they 

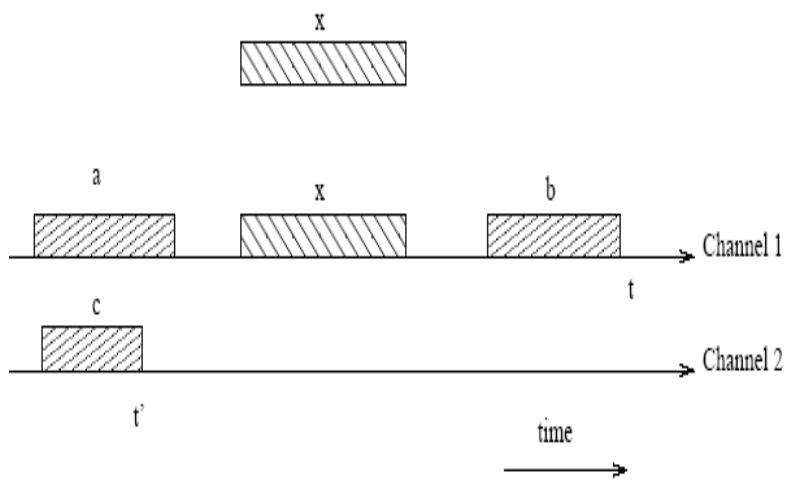

Fig 3: Scheduling by horizon algorithms

consider only one side of a void. Segmented based void filling. Due to this smaller size data bursts may be scheduled in a larger void whereas bigger size data bursts may get blocked. If in general, segment-first schemes will incur lower delays than delay-first schemes.

\subsection{Non-preemptive Delay First Minimum Overlap Channel with Void Filling}

The NP-DFMOC-VF calculates the delay until the first void on every channel and then selects the channel with minimum delay. If a channel is available, the unscheduled burst is scheduled on the free channel with minimum gap. If all channels are busy and the starting time of the first void is greater than or equal to the sum of the end time, Ea, of the unscheduled burst and MAX _DELAY, then the entire unscheduled burst is dropped. Otherwise, the unscheduled burst is delayed until the start of the first void on the selected channel, where the non-overlapping burst segments of the information. The following information is used by the scheduler for all the scheduling algorithms. For example consider the Figure 5, 6 and 7.

- $\quad \mathrm{L}_{\mathrm{b}}$ is the unscheduled burst length duration.

- $\mathrm{t}_{\mathrm{ub}}$ is the unscheduled burst arrival time.

- $\quad \mathrm{ST}$ is switching time required to reconfigure the optical cross-connect at each OBS node.

- D1, D2 and D3 are their respective outgoing data channels. Data burst b1 and b2 are schedule on channel D1, b3 and b4 on channel D2 and b5 and b6 on channel D3.

- $\quad \mathrm{S}\left(\mathrm{i}_{\mathrm{i}, \mathrm{j}}\right)$ and $\mathrm{E}\left(\mathrm{i}_{\mathrm{i}, \mathrm{j}}\right)$ are starting and ending times of each scheduled burst, $j$, on every data channel, $i$, for void filling scheduling algorithms.

- $\mathrm{Gap}_{\mathrm{i}}$ is the difference between $\mathrm{t}_{\mathrm{ub}}$ and $\mathrm{E}\left(\mathrm{i}_{\mathrm{i}, \mathrm{j}}\right)$ of previous schedule burst, $\mathrm{j}$, for scheduling algorithms with void filling.

On channel D1 the end time of data burst b1 is $\mathrm{E}\left({ }_{1,1}\right)$ and start time of data burst b2 is $S(1,3)$. Data burst b3 has end time of $\mathrm{E}_{(2,1)}$ and data burst b4 has start time of $\mathrm{S}_{(2,2)}$ on channel D2. $\mathrm{x}$
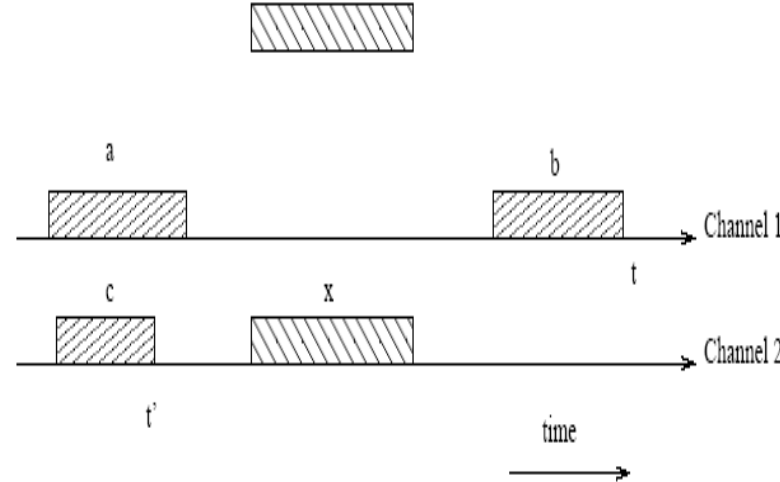

Fig 4: Scheduling by horizon algorithms

Similarly, for data burst b5, $\mathrm{E}\left({ }_{3,1}\right)$ is the end time and for data burst b6, $S\left(_{3,2}\right)$ is the start time. Suppose data burst B1 arrive at a node. The starting time and the ending time of data burst $B 1$ is $S_{a}$ and $E_{a}$ respectively. In the following subsections a unscheduled burst are scheduled, while the overlapping burst segments are dropped. In case the start of the first void is greater than the sum of the start time, Sa, of the unscheduled burst and MAX_DELAY, then the unscheduled burst is delayed for MAX_DELAY and the non-overlapping burst segments of the unscheduled burst are scheduled, while the overlapping burst segments are dropped. For example, consider Figure 5. By applying the NP-DFMOC-VF algorithm, the data channel D1 has the minimum delay, thus the unscheduled burst is scheduled on D1 after delaying the burst using FDLs. In this case, the overlapping segments of the burst are dropped though there is availability of channels D2 and D3 as shown in Figure 5. Though there is presence of channels (D2 and D3) they can be only used for arrival of new bursts, the overlapping segments of the burst B1 are dropped and thus cannot be rescheduled which is the limitations of this algorithm. Hence to overcome this effect we move further to the next algorithm as discussed below.

\subsection{Non-preemptive Segmented First Minimum Overlap Channel with Void Filling (NP-SFMOC-VF)}

The NP-SFMOC-VF algorithm calculates the loss on every channel and then selects the channel with minimum loss. If a channel is available, the unscheduled burst is scheduled on the free channel with minimum gap. If all channels are busy and the starting time of the first void is greater than or equal to the sum of the end time, Ea, of the unscheduled burst and MAX_DELAY, then the entire unscheduled burst is dropped. If the starting time of the first void is greater than or equal to the end time, Ea, of the unscheduled burst, the NP-DFMOCVF algorithm is employed. The unscheduled burst is segmented (if necessary) and the non-overlapping burst segments are scheduled on the selected channel, while the overlapping burst segments are re-scheduled. For the rescheduled burst segments, the algorithm calculates the delay required until the start of the next void on every channel and selects the channel with 


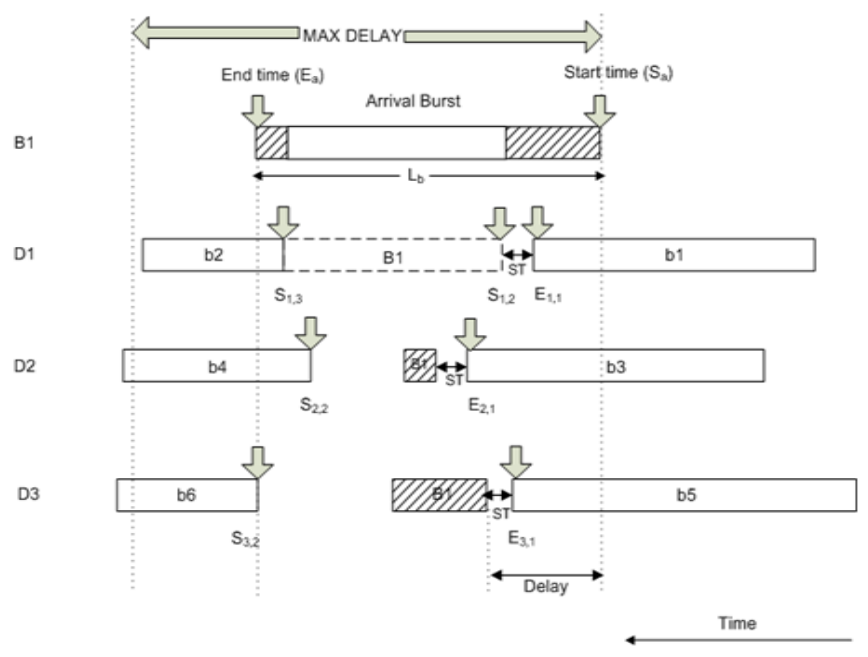

Fig 5: Illustration of NP-DFMOC-VF algorithm

minimum delay. The re-scheduled burst segments are delayed until the start of the first void on the selected channel. The non-overlapping burst segments of the re scheduled burst are scheduled, while the overlapping burst segments are dropped. In case the start of the next void is greater than the sum of the start time, Sa , of the unscheduled burst and MAX_DELAY, the re-scheduled burst segments are delayed for MAX_DELAY and the non-overlapping burst segments of the rescheduled burst are scheduled, while the overlapping burst segments are dropped. For example, in Figure 6, we observe that the data channel D1 has the minimum loss, thus the unscheduled burst is scheduled on D1, and the unscheduled burst B1 has both head overlapping and tail overlapping on which head overlapping re-scheduled burst segments are scheduled on D3 (as it incurs the minimum delay) and tail overlapping rescheduled burst segments are scheduled on D2. Though there is no loss of data bursts as shown in figure but for head overlapping and tail overlapping portion separate channels D3 and D2 respectively has been used which in turns to be expensive in terms of cost and looks un-effective as well. Thus the limitations of existing algorithms are both algorithms consider only one side of void. Next we propose a new channel scheduling algorithms which considers both end of a void in scheduling and also utilizes void efficiency and blocking probability of data burst is minimum.

\section{CHANNEL UTILLIZATION BASED SCHEDULING ALGORITHM}

In this section we introduce a new scheduling algorithm called Best Fit Void Filling (BFVF), which attempts to maximize the channel utilization and minimize the burst loss. Our propose algorithm first selects all possible void channels, on which the data burst can be scheduled. See Figure 7 and 8 Then selects one of the possible void channels such that the void utilization factor is maximum. We calculate the void utilization factor as:

- Utilization $=(\mathrm{a} * 100) / \mathrm{x}$

Where ' $a$ ' is the data burst length and ' $x$ ' is the void length .In Figure 7, for first case, void utilization factor for B1 on channel D1, D2 and D3 are $\left(E_{a}-S_{a}\right) /\left(\left(S_{1,2}\right)-\left(E_{1,1}\right)\right),\left(E_{a}-\right.$

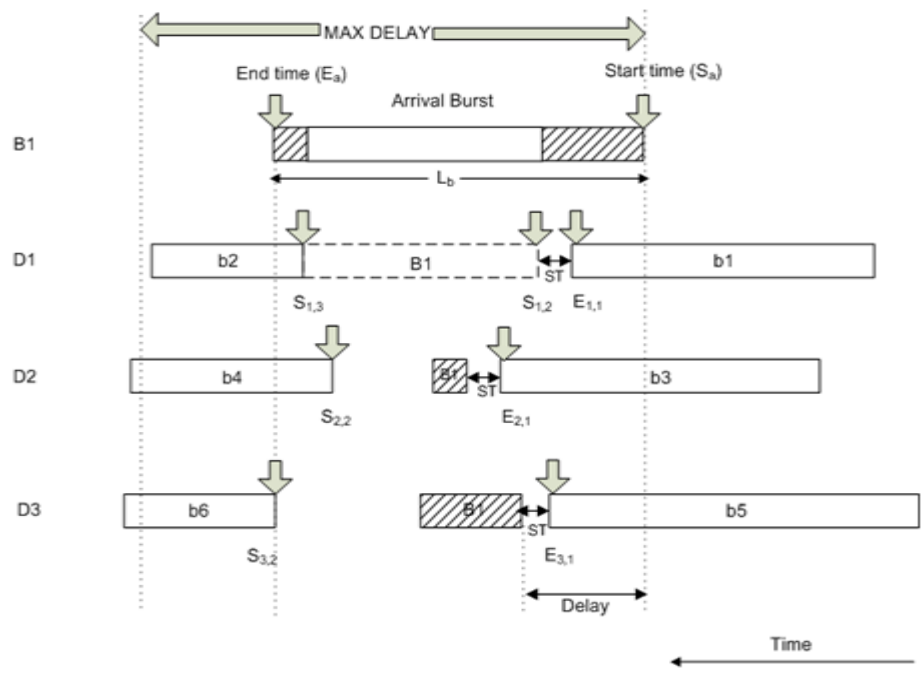

Fig 6: Illustration of NP-SFMOC-VF algorithm

$\left.S_{a}\right) /\left(\left(S_{2,2}\right)-\left(E_{2,1}\right)\right), \quad\left(E_{a}-S_{a}\right) /\left(\left(S_{3,3}\right)-\left(E_{1,1}\right)\right)$ respectively. If void utilization factor exceeds over 100 percent then the factor having close to 100 percent is considered. Here according to figure, using void utilization factor, it selects the channel D3 for the first case to schedule the portion of data burst B1. Since it cannot schedule all the portion of data burst B1 the overlapping portion of data bursts segments is reschedule. For that the remaining channel is D1 and D2 since channel D3 is is already been used. For reschedule data burst segments that is for second case we again calculate the void utilization factor for remaining portion of data burst $\mathrm{B} 1$ which have to be rescheduled and calculated as $\left(\mathrm{E}_{\mathrm{a}}-\mathrm{R}_{\mathrm{a}}\right) /\left(\left(\mathrm{S}_{1,2}\right)-\left(\mathrm{E}_{1,1}\right)\right)$, $\left(\mathrm{E}_{\mathrm{a}}-\right.$ $\left.\mathrm{R}_{\mathrm{a}}\right) /\left(\left(\mathrm{S}_{2,2}\right)-\left(\mathrm{E}_{1,1}\right)\right)$ where $\mathrm{Ra}$ is the start time for reschedule burst segment. In case, the void is greater than MAX_DELAY, the unscheduled burst is delayed for MAX_DELAY and the non overlapping burst segments of unscheduled burst is scheduled, while the overlapping burst segments are dropped. In this case, according to formula the data channel D2 is selected since its channel utilization factor for remaining reschedule burst segment is better than channel D1 (see Figure 7). Hence, the reschedule data burst segment is scheduled on channel D2. And the data channel D1 which is free can be completely used for new arrival data burst. Thus the channel utilization is higher and burst loss ratio is lower in our propose scheme than in NP-DFMOC-VF and NPSFMOC-VF algorithms. We, workout an example to show void utilization on our proposed algorithm. We assume the following numerical values. For first case,

- $\quad\left(\mathrm{S}_{1,2}\right)-\left(\mathrm{E}_{1,1}\right)=(220-140)=80 \mu \mathrm{s}$

- $\quad\left(\mathrm{S}_{2,2}\right)-\left(\mathrm{E}_{2,1}\right)=(210-160)=50 \mu \mathrm{s}$

- $\left(\mathrm{S}_{3,3}\right)-\left(\mathrm{E}_{3,1}\right)=(230-145)=85 \mu \mathrm{s}$

- $\quad$ Length of data burst B1 $\left(\mathrm{L}_{\mathrm{b}}\right)=(\mathrm{Ea}-\mathrm{Sa})=110 \mu \mathrm{s}$

- $\quad$ Switching time $(\mathrm{ST})=10 \mu \mathrm{s}$

- $\quad$ Maximum Delay $=250 \mu \mathrm{s}$

Using channel utilization factor formula,

For D1, channel utilization $=(110 * 100) / 80=137.5 \%$

For D2, channel utilization $=(110 * 100) / 50=220 \%$

For D3, channel utilization $=(110 * 100) / 85=129.4 \%$ 


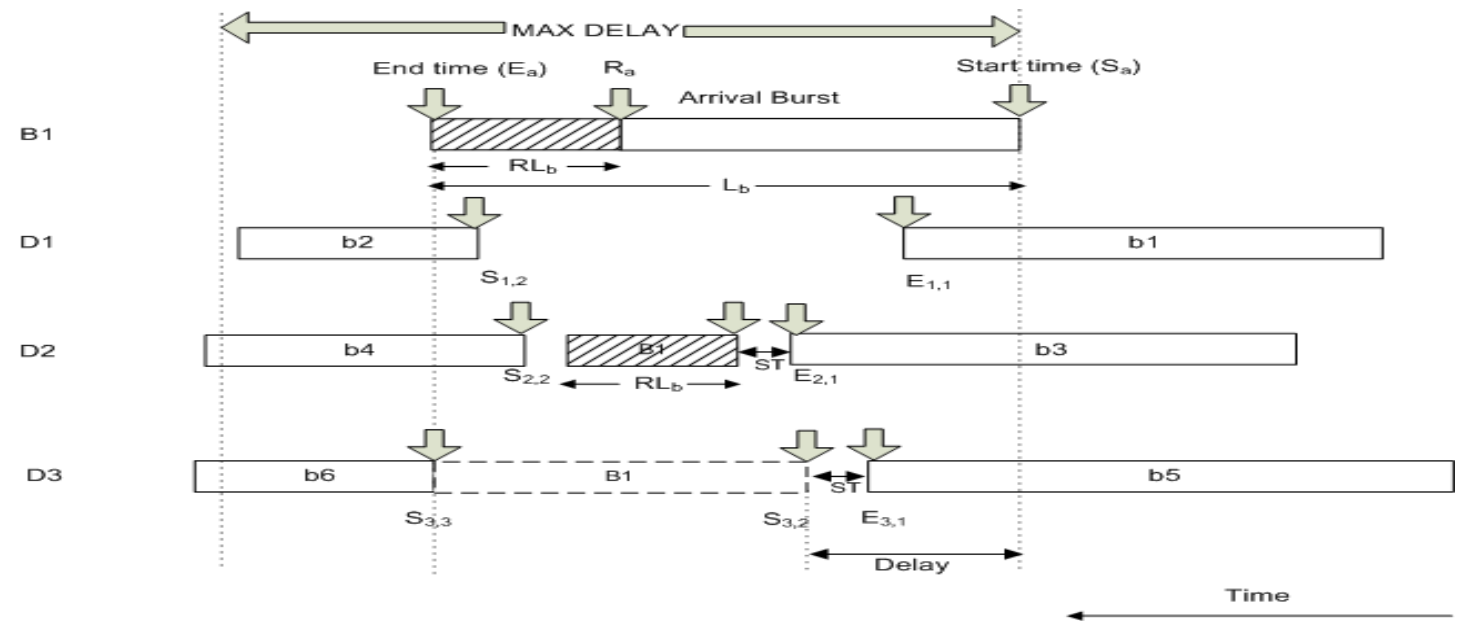

Fig 7: Illustration of Channel Utilization Based Scheduling Algorithm

Table 1. Input data for channel scheduling of different algorithms

\begin{tabular}{|c|c|c|c|c|c|c|c|c|c|}
\hline & \multicolumn{3}{|c|}{ CASE I (NPDFMOC-VF) } & \multicolumn{3}{|c|}{ CASE II (NPSFMOC-VF) } & \multicolumn{3}{|c|}{ CASE III (BFVF SEGMENTED) } \\
\hline & B1 & B2 & B3 & B1 & B2 & B3 & B1 & B2 & B3 \\
\hline $\begin{array}{c}\mathrm{L}_{\mathrm{b}}=\mathrm{Ea}-\mathrm{Sa} \\
(\mu \mathrm{s})\end{array}$ & $90-40=50$ & $137-62=75$ & $102-52=50$ & $135-45=90$ & $140-40=100$ & $129-75=54$ & $230-120=110$ & $275-150=125$ & $270-210=60$ \\
\hline & \multicolumn{3}{|c|}{$(S i, j)-(E i, j) \mu s$} & \multicolumn{3}{|c|}{$(\mathrm{Si}, \mathrm{j})-(\mathrm{Ei}, \mathrm{j}) \mu \mathrm{s}$} & \multicolumn{3}{|c|}{$(S i, j)-(E i, j) \mu s$} \\
\hline D1 & \multicolumn{3}{|c|}{$106-66=40$} & \multicolumn{3}{|c|}{$110-66=44$} & \multicolumn{3}{|c|}{$255-165=90$} \\
\hline D2 & \multicolumn{3}{|c|}{$128-50=78$} & \multicolumn{3}{|c|}{$138-80=58$} & \multicolumn{3}{|c|}{$235-180=55$} \\
\hline D3 & \multicolumn{3}{|c|}{$130-62=68$} & \multicolumn{3}{|c|}{$130-62=68$} & \multicolumn{3}{|c|}{$240-200=40$} \\
\hline D4 & \multicolumn{3}{|c|}{$135-70=65$} & \multicolumn{3}{|c|}{$135-75=60$} & \multicolumn{3}{|c|}{$220-140=80$} \\
\hline D5 & \multicolumn{3}{|c|}{$140-75=65$} & \multicolumn{3}{|c|}{$120-85=35$} & \multicolumn{3}{|c|}{$210-160=50$} \\
\hline D6 & \multicolumn{3}{|c|}{$110-72=38$} & \multicolumn{3}{|c|}{$110-70=40$} & \multicolumn{3}{|c|}{$230-145=85$} \\
\hline D7 & & & & \multicolumn{3}{|c|}{$130-50=80$} & \multicolumn{3}{|c|}{$255-210=45$} \\
\hline D8 & & & & \multicolumn{3}{|c|}{$135-90=45$} & \multicolumn{3}{|c|}{$250-215=35$} \\
\hline D9 & & & & \multicolumn{3}{|c|}{$140-85=55$} & \multicolumn{3}{|c|}{$252-220=32$} \\
\hline ST $(\mu \mathrm{s})$ & \multicolumn{3}{|c|}{10} & \multicolumn{3}{|c|}{10} & \multicolumn{3}{|c|}{10} \\
\hline W & \multicolumn{3}{|c|}{6} & \multicolumn{3}{|c|}{9} & \multicolumn{3}{|c|}{9} \\
\hline $\begin{array}{l}\text { Maximum } \\
\text { Delay }(\mu s)\end{array}$ & & 127 & & & 200 & & & 255 & \\
\hline
\end{tabular}


Table 2. Output data for channel scheduling of different algorithms

\begin{tabular}{|c|c|c|c|c|c|c|c|c|c|}
\hline & \multicolumn{3}{|c|}{ CASE I } & \multicolumn{3}{|c|}{ CASE II } & \multicolumn{3}{|c|}{ CASE III } \\
\hline & $\begin{array}{c}\text { NPDFMOC } \\
-V F\end{array}$ & $\begin{array}{c}\text { NPSFMOC } \\
-V F\end{array}$ & $\begin{array}{c}\text { BFVF } \\
\text { Segmented } \\
\text { Based }\end{array}$ & $\begin{array}{c}\text { NPDFMOC } \\
-V F\end{array}$ & $\begin{array}{c}\text { NPSFMOC } \\
-V F\end{array}$ & $\begin{array}{c}\text { BFVF } \\
\text { Segmented } \\
\text { Based }\end{array}$ & $\begin{array}{c}\text { NPDFMOC } \\
-V F\end{array}$ & $\begin{array}{c}\text { NPSFMOC } \\
-V F\end{array}$ & $\begin{array}{c}\text { BFVF } \\
\text { Segmented } \\
\text { Based }\end{array}$ \\
\hline $\begin{array}{c}\text { Delay for } \\
\text { non } \\
\text { overlapping } \\
\text { burst B1 }\end{array}$ & $20 \mu \mathrm{s}$ & $20 \mu \mathrm{s}$ & $40 \mu \mathrm{s}$ & $27 \mu \mathrm{s}$ & $27 \mu \mathrm{s}$ & $25 \mu \mathrm{s}$ & $30 \mu \mathrm{s}$ & $30 \mu \mathrm{s}$ & $55 \mu \mathrm{s}$ \\
\hline $\begin{array}{c}\text { Delay for } \\
\text { non } \\
\text { overlapping } \\
\text { burst B2 }\end{array}$ & $10 \mu \mathrm{s}$ & $10 \mu \mathrm{s}$ & $10 \mu \mathrm{s}$ & $36 \mu \mathrm{s}$ & $45 \mu \mathrm{s}$ & $32 \mu \mathrm{s}$ & $5 \mu \mathrm{s}$ & $25 \mu \mathrm{s}$ & $5 \mu \mathrm{s}$ \\
\hline $\begin{array}{c}\text { Delay for } \\
\text { non } \\
\text { overlapping } \\
\text { burst B3 }\end{array}$ & $24 \mu \mathrm{s}$ & $30 \mu \mathrm{s}$ & $33 \mu \mathrm{s}$ & $15 \mu \mathrm{s}$ & $25 \mu \mathrm{s}$ & $15 \mu \mathrm{s}$ & $0 \mu \mathrm{s}$ & $10 \mu \mathrm{s}$ & $0 \mu \mathrm{s}$ \\
\hline $\begin{array}{c}\text { Number of } \\
\text { channel } \\
\text { Used }\end{array}$ & 3 & 6 & 4 & 3 & 9 & 6 & 3 & 9 & 6 \\
\hline $\begin{array}{c}\text { Total packet } \\
\text { loss }\end{array}$ & $30 \mu \mathrm{s}$ & $0 \mu \mathrm{s}$ & $0 \mu \mathrm{s}$ & $10 \mu \mathrm{s}$ & $0 \mu \mathrm{s}$ & $0 \mu \mathrm{s}$ & $115 \mu \mathrm{s}$ & $0 \mu \mathrm{s}$ & $2 \mu \mathrm{s}$ \\
\hline
\end{tabular}

Here, we select the channel D3 since channel utilization of channel D3 is close to 100 percent as compare to channel D1 and D2. Note if the channel utilization had been less than 100 percent we go for channel utilization less than 100 percent instead of more than 100 percent. For second case, (for $\mathrm{RL}_{\mathrm{b}}$ )

Length of remaining data burst segment of $\mathrm{B} 1,\left(\mathrm{RL}_{\mathrm{b}}\right)$

- $\mathrm{RL}_{\mathrm{b}}=($ Ea-Ra $)=230-195=35 \mu \mathrm{s}$

Remaining channel D1 and D2

- $\quad\left(\mathrm{S}_{1,2}\right)-\left(\mathrm{E}_{1,1}\right)=(220-140)=80 \mu \mathrm{s}$

- $\quad\left(\mathrm{S}_{2,2}\right)-\left(\mathrm{E}_{2,1}\right)=(210-160)=50 \mu \mathrm{s}$

- $\quad$ Switching time $(\mathrm{ST})=10 \mu \mathrm{s}$

Using channel utilization factor formula for $\mathrm{RL}_{\mathrm{b}}$,

For D1, channel utilization $=(35 * 100) / 80=43 \%$

For D2, channel utilization $=(35 * 100) / 50=70 \%$

In this case, channel D2 is selected for reschedule the remaining data burst of $\mathrm{B} 1$ i.e for $\mathrm{RL}_{\mathrm{b}}$. Also, the free channel $\mathrm{D} 1$ can be used for new arrival of data bursts. This shows that void utilization is higher in our proposed algorithm.

\section{SIMULATION AND RESULTS}

We compare the performance of our introduced BFVF segmented based algorithm with that of NP-DFMOC-VF and
NP-SFMOC-VF algorithm through simulation. For simulation proposed and to be more précised we take three cases for channel scheduling. In each case we take three bursts B1, B2 and B3 which have to be scheduled by using different algorithms. $\mathrm{W}$ is the maximum number of outgoing data channels. According to given input data of table I, we obtained an output as Table 1 which is shown below. Considering a Table 2 and its cases I, II and III we can see that in Case I delay is more in our proposed algorithm as compare to NP-DFMOC-VF and NP-SFMOC-VF but in Case II delay is less in our proposed algorithm than NP-DFMOCVF and NP-SFMOC-VF where as in Case III in our proposed algorithm delay is more for data burst $\mathrm{B} 1$ and less for data burst B2 and B3 as compare to NP-DFMOC VF and NPSFMOC-VF. Hence we can say that delay does not depend on type of algorithm we used but it depends on how the data bursts are schedule on the channels. Also from simulation of Figure 8-10 this can be seen. Again considering table 2, this time we consider total packet loss for different algorithms versus number of channel used for different algorithms. According to table we simulate the result for this as shown in Figure 11, 12 and 13. We can see that packet loss for our proposed algorithm is zero for case I and II and in case III packet losses are very low and number of channel used is also less comparing to NPSFMOC-VF algorithm. In NPDFMOC$\mathrm{VF}$ algorithm, though the number of channel used is less than NPSFMOC-VF and our proposed algorithm but the packet losses are very high in NPDFMOC-VF then NPSFMOC-VF and our proposed algorithm. 


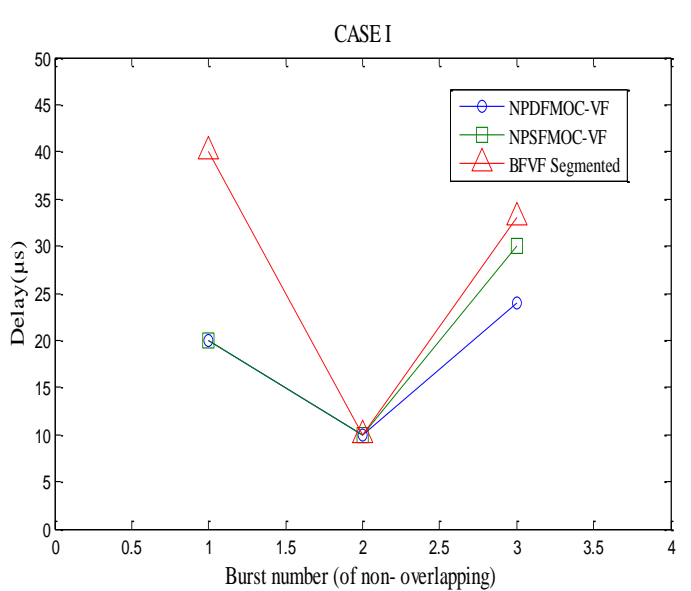

Fig 8: Delay vs. non overlapping burst for case I

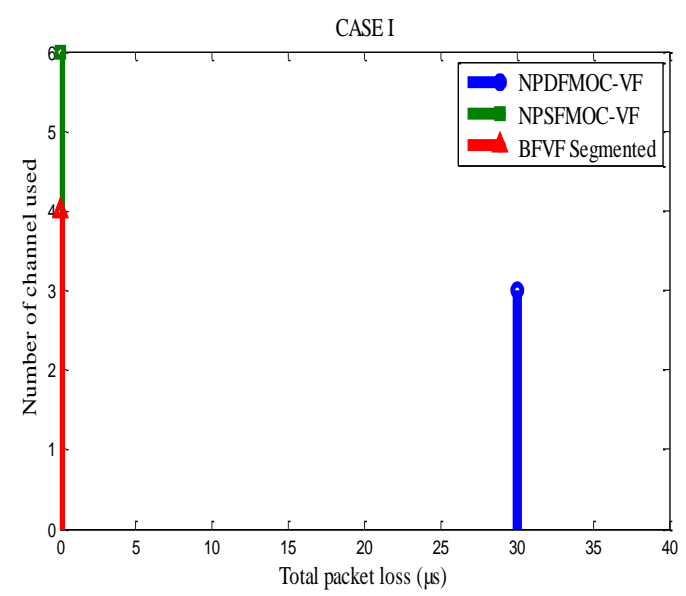

Fig 11: Number of channel used vs. Total packet loss for case I

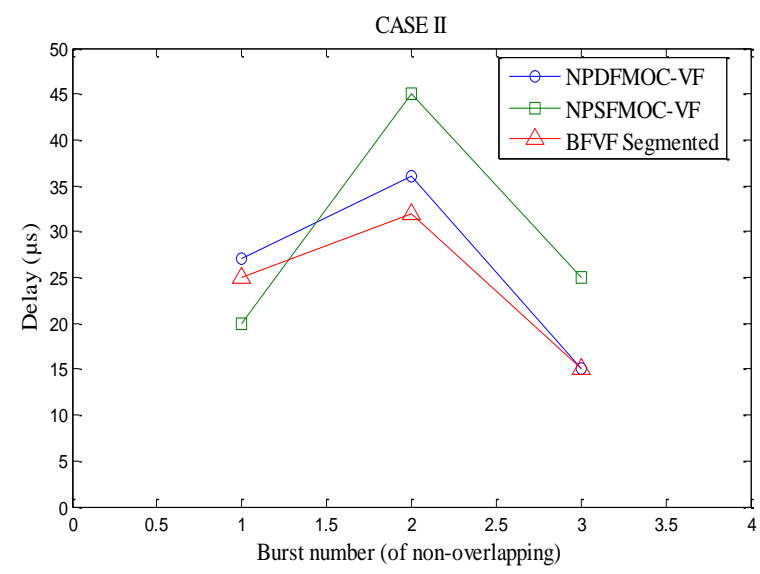

Fig 9: Delay vs. non overlapping burst for case II

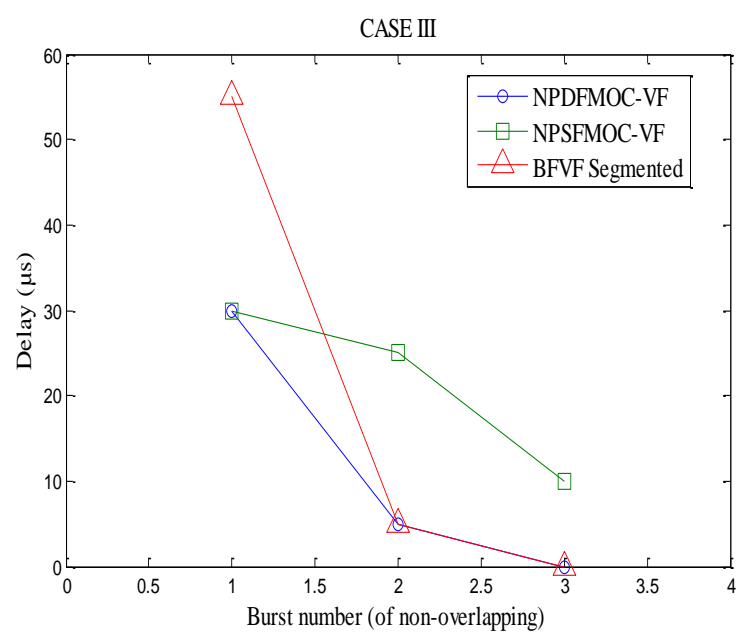

Fig 10: Delay vs. non overlapping burst for case III

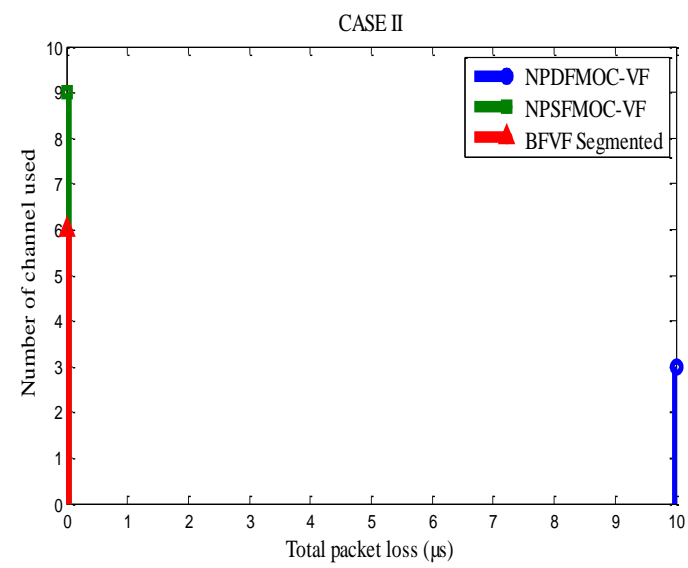

Fig 12: Number of channel used vs. Total packet loss for case II

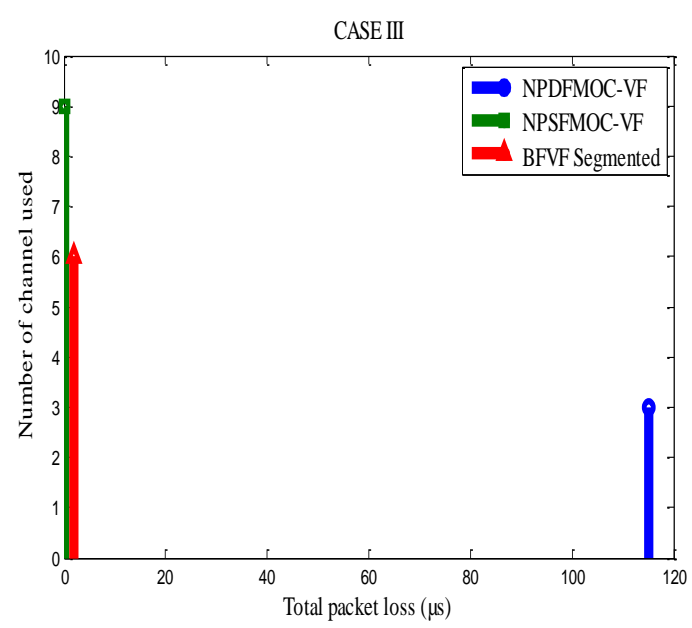

Fig 13: Number of channel used vs. Total packet loss for case III 
Also from Figure 8-10 we draw a table and conclude the comparison of burst loss and channel utilization as follows.

Table 3. Comparisons of different algorithm in terms of Burst Loss and Channel Utilization

\begin{tabular}{|c|c|c|}
\hline Algorithm & Burst Loss & Channel Utilization \\
\hline NPDFMOC-VF & High & High \\
\hline NPSFMOC-VF & Low & Low \\
\hline BFVF Segmented & Low & High \\
\hline
\end{tabular}

\section{CONCLUSION}

In this paper comparative analysis of existing void filling and non void filling schemes of channel scheduling in core node of an OBS network $g$ has been done. Thereafter a new scheme based on channel utilization for scheduling in core node has been introduced. It is found that the newly introduced scheme performs better than the horizon scheduling algorithms. However, there are limitations to the existing void filling scheduling algorithms. This limitation is mainly due to that; the existing schemes consider either the start time of the new data burst and end time of the previously scheduled data burst or start time of previously scheduled data burst and the end time of the new data burst. They do not take into account the data burst length and void length. We introduced a new scheme called BFVF Segmented based algorithm, which takes the arrival data burst length and void length into account in scheduling. Proposed scheme calculates the void utilization factor, and schedule the new data burst into a void channel having maximum void utilization factor. The newly introduced scheme is compared with NPDFMOCVF and BFVF Segmented. It is found that the newly introduced scheme perform better in terms of channel utilization, packet loss and number of channel used.

\section{REFERENCES}

1] K. Koduru 2005 New Contention Resolution Techniques for Optical Burst Switching, Master's thesis, Louisiana State University.

[2] V. M Vokkarane and J.P Jue. 2003 Segmentation-based non preemptive scheduling algorithms for optical burstswitched networks. IEEE First Inter-national Workshop on Optical Burst Switching (WOBS)

[3] K. Dolzer and C. Gauger, 2007 On burst assembly in optical burst switching networks - a performance evaluation of Just-Enough-Time", Proceedings of the 17th International Teletraffic Congress (ITC 17), Salvador

[4] Y. Xiong, M. Vandenhoute, and H. C. Cankaya, 2000. Control Architecture in Optical Burst Switched WDM Networks. IEEE JSAC, vol. 18, no. 10, pp. 1838 - 1851.

[5] M. Yoo, C. Qiao, and S. Dixit, 2000 QoS Performance of Optical Burst Switching in IP-Over-WDM Networks, IEEE Journal on Selected Areas in Communications, vol. 18.

[6] W. M. Golab and R. Boutaba, 1998 Resource Allocation in User-Controlled Circuit-Switched Optical Networks, LNCS Spinger- Verlag, vol. 16, no. 12, 2081 - 2094, December 1998.

[7] M. Yang, S. Q. Zheng, and D. Verchere, 2001 A QoS Supporting Scheduling Algorithm for Optical Burst Switching DWDM Networks, GLOBECOM 0186 - 91.

[8] L. Xu, H.G. Perros, and G. Rouskas, 2001 Techniques for optical packet switching and optical burst switching, IEEE Communications Magazine, Vol. 39, 136-142.

[9] V.M. Vokkarane, G.P.V. Thodime, V.B.T. Challagulla, and J.P. Jue.2008 Channel scheduling algorithms using burst segmentation and FDLs for optical burst-switched networks IEEE ICC.

[10] M. Yoo, M. Jeong and C. Qiao, 2007 A High Speed Protocol for Bursty Traffic in Optical Networks", in SPIE's All-Optical Communication Systems: Architecture, Control and Protocol Issues, Vol. 3230, 79-90.

[11] Aydin, M.A.; Atmaca, T.; Turna, O.C.; Zaim, H., 2009 Performance Study of New OBS Channel Scheduling Algorithms in a Multiservice Network. etworking and Services, 2009. ICNS '09. Fifth International Conference. 242- 248

[12] Jinhui Xu; Chunming Qiao; Li, J.; Guang Xu, : 2004 Efficient burst scheduling algorithms in optical burstswitched networks using geometric techniques. Selected Areas in Communications, IEEE Journal Volume:22 , Issue: 9, 1796- 1811.

[13] Hongyun Zheng; Changjia Chen; Yongxiang Zhao, 2006 Optimization Scheduling for Optical Burst Switching Networks with Wavelength Conversion. Communication Technology, 2006. ICCT '06. International Conference 14.

[14] R Netak, L.D.; Chowdhary, G.V.; Suryawanshi, V.N.; Borade, J.2011 Reverse scheduling approach for burst loss minimization in WDM OBS based networks.Computer and Communication Technology (ICCCT), 2011 2nd International Conference. 517 523 